\title{
A Method to Compute Flameout Limits of Scramjet-Powered Hypersonic Vehicles
}

\author{
Chukwuka C. Mbagwu*, James F. Driscoll ${ }^{\dagger}$
}

\begin{abstract}
As a hypersonic vehicle travels upward along an ascent trajectory, the static pressure $\left(p_{3}\right)$ in the scramjet combustor will decrease, which can lead to engine flameout. At low pressures the chemical reactions between the fuel and air become excessively slow. However, during the ascent the flight Mach number is increasing; this increases the stagnation temperature and the static temperature $\left(T_{3}\right)$ at the combustor entrance so it tends to prevent flameout. To investigate this tradeoff, a general method to understand how the flameout limit varies during ascent was developed. The method consists of two parts; first the static temperature and pressure at the entrance to the combustor $\left(T_{3}, p_{3}\right)$ are computed as a function of the vehicle altitude; this is done using a reduced-order propulsion model called MASIV. In the second part the values of $\left(T_{3}, p_{3}\right)$ are inserted into an empirical relation for the critical Damkohler number in order to determine if flameout occurs or not at each altitude. The empirical flameout relation is based on previous ground-based measurements made at AFRL and elsewhere.
\end{abstract}

\section{Introduction}

Consider the hypersonic wave rider that is shown in Figure 1. This vehicle, called MAX-1, ascends along the constant dynamic pressure trajectory (the solid line) in Figure 2, which is a plot of altitude versus flight Mach number. It is desired to compute a second curve that is the engine flameout operability limit; this limit is sketched as the dotted line in Figure 2. The trajectory curve then can be modified so as to avoid the flameout limit curve. For example, in a dual-mode ramjet-scramjet engine it is known that whenever the static pressure at the combustor entrance becomes too small (typically less than $0.5 \mathrm{~atm}$.) then flameout is possible. However, static pressure is only one of several parameters that govern flameout.
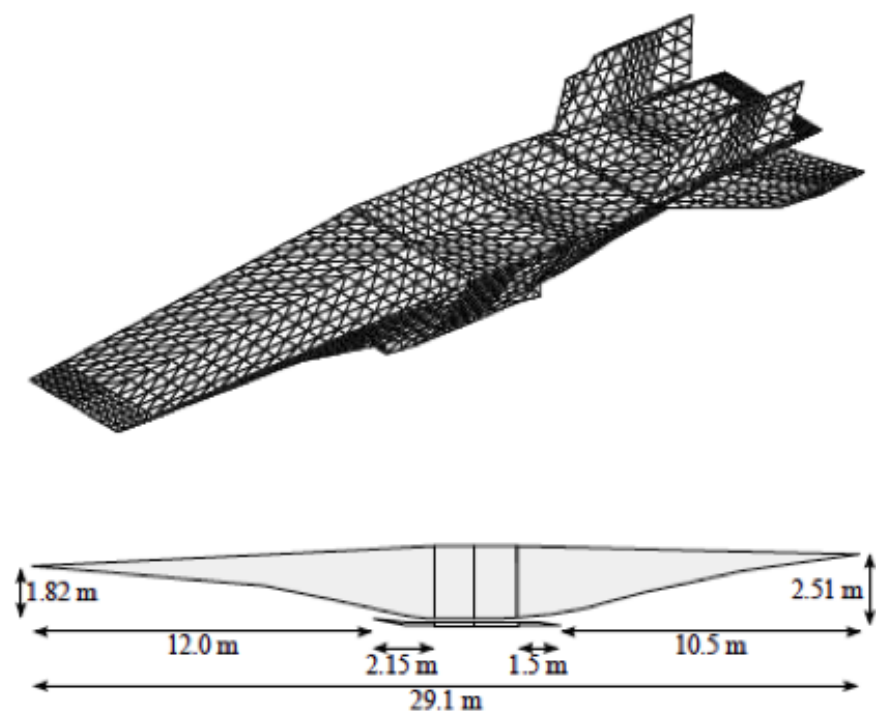

Figure 1. MAX-1 vehicle and flow path dimensions. Engine width is $2.143 \mathrm{~m}$.

The solid curve in Figure 2 represents a constant dynamic pressure $\left(q=\frac{1}{2} \rho_{\infty} U_{\infty}^{2}=100 \mathrm{kPa}\right)$ trajectory. As the vehicle flight Mach number increases from 4.4 to 7.0 along this trajectory, the altitude must increase from $18 \mathrm{~km}$ to $24 \mathrm{~km}$. It can be shown that for a constant $q$ ascent trajectory, the ambient pressure $\left(p_{\infty}\right)$ will decrease and is

${ }^{*}$ Ph.D. Candidate, Dept. of Aerospace Engineering, Univ. of Michigan, USA, AIAA Member, cmbagwu@umich . edu

${ }^{\dagger}$ Professor, Dept. of Aerospace Engineering, Univ. of Michigan, USA, AIAA Fellow, james fd@umich.edu 
proportional to $\left(1 / M_{\infty}^{2}\right)$. For the special case of an inlet that provides a constant compression ration $\left(p_{3} / p_{\infty}\right)$, it follows that combustor pressure $p_{3}$ will also be proportional to $\left(1 / M_{\infty}^{2}\right)$. Thus, increasing the Mach number from 4.4 to 7.0, as sketched in Figure 2, leads to a nearly threefold increase in the combustor pressure. This can cause such a large decrease in the chemical reaction rate of the fuel chemistry that the result is engine flameout.

The dotted line in Figure 2 is a sketch of a possible flame out limit of the vehicle; this curve is derived in section 7. It is not a general result but rather depends on the specific geometry of the flame holder and the engine flow path. It has a shape that shows that flying at excessively high altitudes leads to flameout due to very small combustor pressures. Also, as flight Mach number increases, the stagnation temperature becomes so large that the air static temperature exceeds the auto-ignition temperature. A flame holder is no longer necessary, but flameout can still occur if the ignition delay time exceeds the residence time that the fuel-air mixture spends in the combustor section.

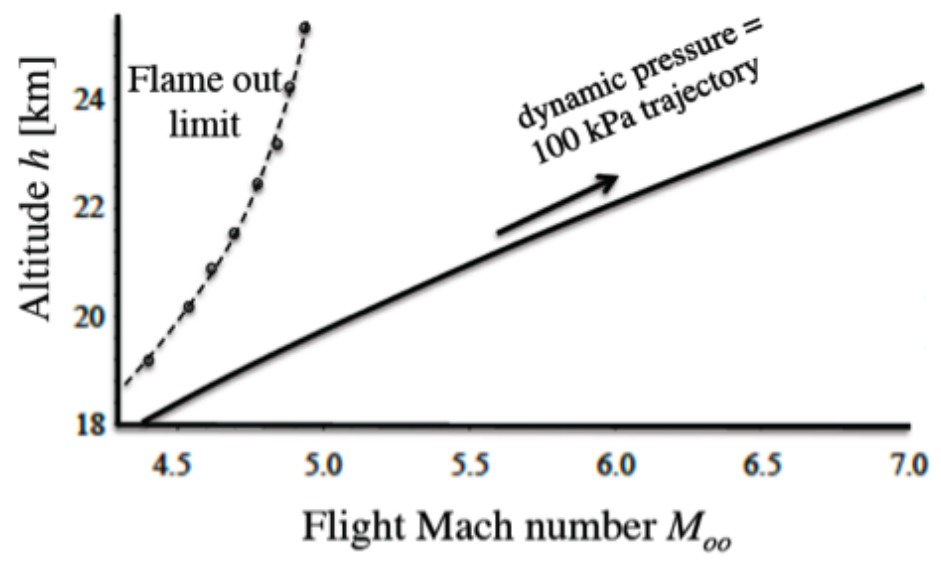

Figure 2. Schematic of a possible flameout limit (dotted line), and a selected vehicle ascent trajectory (solid line, q = $100 \mathrm{kPa}$ ).

\section{Approach}

In order to compute the flameout limit, it is necessary to first assume that a wall-cavity flame holder is employed, and then use an empirical relation for the measured critical Damkohler number at flameout. This approach has been used to estimate flameout limits in afterburners, using the Ozawa empirical flameout relation [1] or a similar empirical relation [2-6]. Damkohler number $[1,4,7]$ is an important propulsion parameter that is defined to be:

$$
D a_{H}=\frac{R R_{F}\left(E R, p_{3}, T_{3}\right)}{U_{3} / H}
$$

The characteristic length scale is the height $(H)$ of the wall-cavity. Cavity flame holders have been studied extensively [7-12]. $R R_{F}$ is the maximum reaction rate of the fuel (in $1 /$ seconds) that is defined in Section 5 below. It depends on the equivalence ration $(E R)$, static pressure $\left(p_{3}\right)$ and static temperature $\left(T_{3}\right)$. Station 3 is defined to be the combustor entrance; station 1 is the leading edge of the vehicle and station 2 is the entrance to the constant-area isolator portion of the inlet (as shown in Figure 3). $U_{3}$ is the air velocity at the combustor entrance.

The critical value of Damkohler number $\left(D a_{H}^{*}\right)$ that causes flameout must be determined from experiments because no CFD computations can reliably compute this value. Fortunately there are many measured values for a cavity flame holder already available. Next the quantity $U_{3}^{*}$ ) is defined; it is the maximum air velocity at the combustor entrance that is allowed before flameout will occur. A formula for $U_{3}^{*}$ is determined by rearranging equation (1):

$$
U_{3}^{*}=\frac{R R_{F}}{D a_{H}^{*}} H
$$

As the vehicle ascends, the air velocity $U_{3}$ at the combustor entrance will increase. When the velocity $U_{3}$ equals the right side of equation (2), flameout occurs. So, the flameout limit is designed to be:

$$
U_{3}=U_{3}^{*}
$$

Therefore, equations (2) and (3) are the primary relations that are used to compute the flameout limit. However, the right side of equation (2) changes during ascent and must be computed at each altitude along the trajectory. The reason 
why $U_{3}$ increases during the ascent is that the increase in the free stream Mach number causes the inlet stagnation temperature to increase. This raises the static temperature $T_{3}$ at the combustor entrance, as well as the local speed of sound, and this tends to increase $U_{3}$.

\section{Previous Related Work}

This section is a brief summary of previous reduced-order models (ROMs) of hypersonic vehicles. A reduced order model is one that rapidly accesses pre-computed lookup tables; the lookup tables were generated using CFD codes for one particular geometry or for the chemical kinetics of one type of fuel.

In 2006, the Bolender-Doman AFRL model $[13,14]$ simulated the flight dynamics of a hypersonic vehicle but it neglected many details of the propulsion system. To improve the AFRL model, a joint effort between the University of Michigan and AFRL led to the MASIV (Michigan-AFRL Scramjet In-Vehicle) second-generation model [1519]. Items that were added include multiple interacting shock waves in the 2-D inlet (and the exhaust nozzle) using a technique similar to the method of characteristics and 3-D fuel-air mixing. 3-D mixing was added by included experimentally-measured formulas for the variation of mixture fraction within a fuel jet that is injected into an air cross-flow. Also added was finite-rate chemistry, by including strained flamelet lookup tables similar to those used in the CFD code FLUENT. A trim code was added to compute lift, drag and moments in order to trim the vehicle at the proper angle of attack at each altitude during the ascent. This work led to a series of publications, one of which demonstrated that the MASIV code can compute the optimum acceleration time-history that minimizes the fuel required for ascent [15]. MASIV also was used to compute the ram-scram transition boundary [15] and the operability limit where the initial stages of engine unstrap occurs. Other reduced-order models of hypersonic vehicles have been reported by O'Neill and Lewis [20]. Bowcutt [21] and others [22-26] developed models for untrimmed vehicles that are operated far from the flameout limit. However, no previous reduced-order model has attempted to compute flameout. So the new aspects of this work are that it pro ides a methodology for computing the engine flameout limits, and the methodology can be applied to a trimmed vehicle undergoing an ascent trajectory to generate operability limits, such as the dotted line in Figure 2.

There is a strong motivation to develop reduced-order models for hypersonic vehicles. A ROM provides an approximate solution rapidly; they are commonly used in the design of a control algorithm or to obtain a first estimate of an optimal aircraft design. A ROM also gives a "first look" at a large parameter space. The ROM can identify certain optimal conditions; at a later time, relatively few high-fidelity CFD runs then can be performed. The MASIV code computes aerodynamic forces and engine thrust for the MAX-1 wave rider by solving ordinary differential equations in less than five seconds on a single $2.6 \mathrm{GHz}$ processor. With multiple processors the computational time drops to a fraction of a second.

Suppose that it is desired to compute the aircraft forces, moments, and the trimmed angle of attack at each point along six possible trajectories to determine the optimum trajectory. Forces on the vehicle must be computed approximately 1800 times. This is because for each trajectory the vehicle must be trimmed at approximately twenty different altitudes. At each altitude the forces have to be computed about fifteen times to determine (by iteration) what angle of attack, equivalence ratio, and elevator settings lead to the trim of the vehicle. A ROM does not compete against high-fidelity CFD, but instead complements it. The full CFD solution is more accurate than the reduced-order model solutions, but CFD is not appropriate early in the design cycle, where thousands of combinations of flight conditions and control inputs are needed for quick performance analysis on ascent trajectories, vehicle design optimization, and control applications.

Two disadvantages of a reduced-order model are that the level of accuracy of the ROM must be determined by validation studies, and the ROM is only valid for the one specific geometry for which the lookup tables were generated. In this case, only quasi-steady changes are considered. There are two types of ROMs: one interpolates between fulloder CFD solution snapshots, and the other is used here in MASIV. MASIV is a first-principles ROM that solves the fundamental conservation equations. While 2-D or 1-D assumptions are employed, still it is possible to investigate the physics to better understand any interesting model predictions.

\section{The MAX-1 Hypersonic Vehicle}

The MAX-1 wave rider is drawn in Figure 1; it is similar to the generic aircraft that was first considered by Bolender and Doman [13]. It has a length of $29.1 \mathrm{~m}$ and the width of the dual mode ramjet-scramjet is $2.143 \mathrm{~m}$. The inlet is rectangular with a sufficiently large aspect ration of 15.3 such that it can be considered to be two-dimensional. The isolated is $1.38 \mathrm{~m}$ long and is folioed by the constant area portion of the combustor that is $0.90 \mathrm{~m}$ long. Both have a cross section of $0.14 \mathrm{~m}$ by $2.143 \mathrm{~m}$. The second part of the combustor is $0.62 \mathrm{~m}$ and it's upper wall diverges at 4 degrees. 
In previous studies the sizes of the control surfaces were optimized, the weight distribution altered, and aero-elastic properties were added [14-19]. Forces on each surface panel are computed using a small-angle panel method and the method to trim the vehicle is described in [15]. The engine inlet is drawn in Figure $1 \mathrm{~b}$ and it contains multiple shock waves that interact. A code called SAMURI, described in [16], is used to compute the static pressure rise and the stagnation pressure loss in the inlet and exhaust nozzle. It assumes that the flow is 2-D, wall deflection angles are small, no separation occurs, and that the supersonic inlet Mach number is small enough that strong shock/boundary layer interactions do not occur.

\section{MASIV Combustor Model}

The MASIV combustor code was described in detail in [17]. This code includes finite-rate chemistry, real gas properties, a three-dimensional jet mixing model, a separated boundary layer model, and gas dissociation. The air stream is modeled as a 1-D flow in the duct drawn in Figure 3. It has variable area, friction, wall heat transfer and head addition due to combustion. A 3-D pattern of fuel jets in a cross flow is superimposed on this 1-D flow. Fuel is injected from 30 ports that are located at one $\mathrm{x}$-location that is $0.14 \mathrm{~m}$ downstream of station 3 in Figure 3 . The 30 fuel jets are located at different span-wise locations across the $2.143 \mathrm{~m}$ width; each port is $3.45 \mathrm{~cm}$ in diameter. Each port is choked and the hydrogen fuel enters the combustor at $300 \mathrm{~K}$ at the sonic speed of $1295 \mathrm{~m} / \mathrm{s}$.

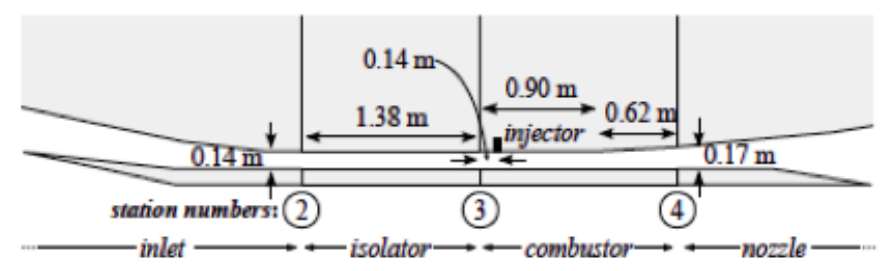

Figure 3. Dual-mode ramjet-scramjet internal flow path of MAX-1 wave rider.

MASIV solves the following seven ordinary differential equations, which include the conservation of mass (4), momentum (5), energy (9), and species (10). The equations are derived in [7].

$$
\begin{gathered}
\frac{1}{\rho} \frac{d \rho}{d x}=\frac{1}{\dot{m}} \frac{d \dot{m}}{d x}-\frac{1}{u} \frac{d u}{d x}-\frac{1}{A} \frac{d A}{d x} \\
\frac{1}{u} \frac{d u}{d x}=-\frac{1}{\rho u^{2}} \frac{d p}{d x}-\frac{2 c_{f}}{D}+\left(1-\frac{u_{F}}{u}\right) \frac{1}{\dot{m}} \frac{d \dot{m}}{d x} \\
\frac{1}{p} \frac{d p}{d x}=\frac{1}{\rho} \frac{d \rho}{d x}+\frac{1}{T} \frac{d T}{d x}-\frac{1}{W} \frac{d W}{d x} \\
\frac{1}{W} \frac{d W}{d x}=-\sum_{i=1}^{n_{s p}} \frac{W}{W_{i}} \frac{d Y_{i}}{d x} \\
\frac{d \dot{m}}{d x}=\sum_{i=1}^{n_{s p}} \frac{d \dot{m}_{i, F}}{d x} \\
c_{p} \frac{d T}{d x}=\frac{h_{0, F}-h_{0}}{\frac{\dot{m}}{d x}}-\frac{2 c_{f} c_{p}\left(T_{a w}-T_{w}\right)}{\operatorname{Pr}^{2 / 3} D}-u \frac{d u}{d x}-\sum_{i=1}^{n_{s p}} h_{i} \frac{d Y_{i}}{d x} \\
\frac{d Y_{i}}{d x}=\frac{\bar{\omega}_{i} W_{i} A}{\dot{m}}+\frac{1}{\dot{m}} \frac{d \dot{m}_{i, F}}{d x}-\frac{Y_{i}}{\dot{m}} \frac{d \dot{m}}{d x}
\end{gathered}
$$

Equations (6) and (7) define the equation of state and the molecular weight $(W)$ of the gas mixture, respectively. Equation (8) states that the total mass flow rate is that of the air plus injected fuel.

An important quantity is $\bar{\omega}_{i}$ that appears in equation (10). It is the volumetric reaction rate of each species, in moles $/ \mathrm{sec} / \mathrm{m}^{3}$. The bar denotes that it has been averaged over the $\mathrm{y}$ and $\mathrm{z}$ directions so that it is only a function of the stream wise coordinate $\mathrm{x}$. To compute the volumetric reaction rate $\bar{\omega}_{i}$, a 2-D empirical sub-model for the fuel-air 
mixing and combustion was developed, because mixing cannot be properly simulated in one dimension. The 3-D mixing model is described in [27]. First it is assumed that the fuel is injected as a jet in a cross-flow. Experiments show that the centerline of the fuel jet bends over such that its y-coordinate $\left(y_{C}\right)$ is proportional to $\left(x_{C}\right)^{1 / 3}$, as given in [17-19]:

$$
\frac{y_{C}}{d_{F}}=c_{2}\left(\frac{x_{C}}{d_{F}}\right)^{1 / 3} R^{2 / 3}
$$

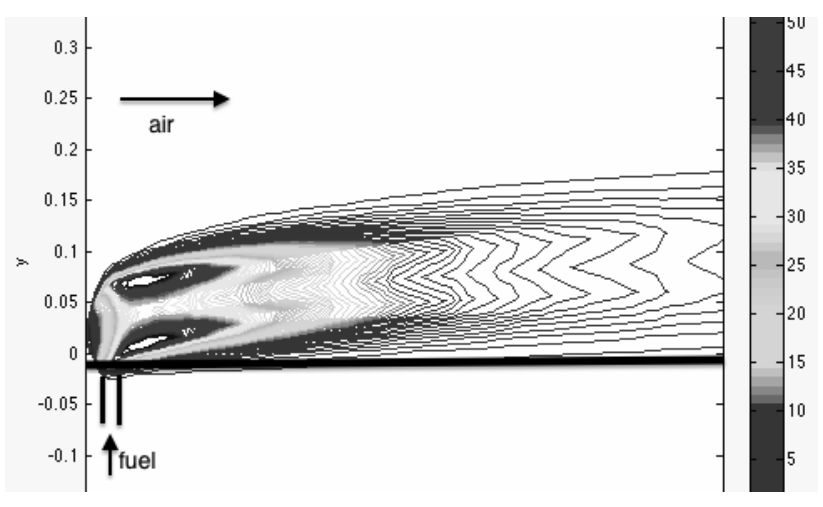

a)

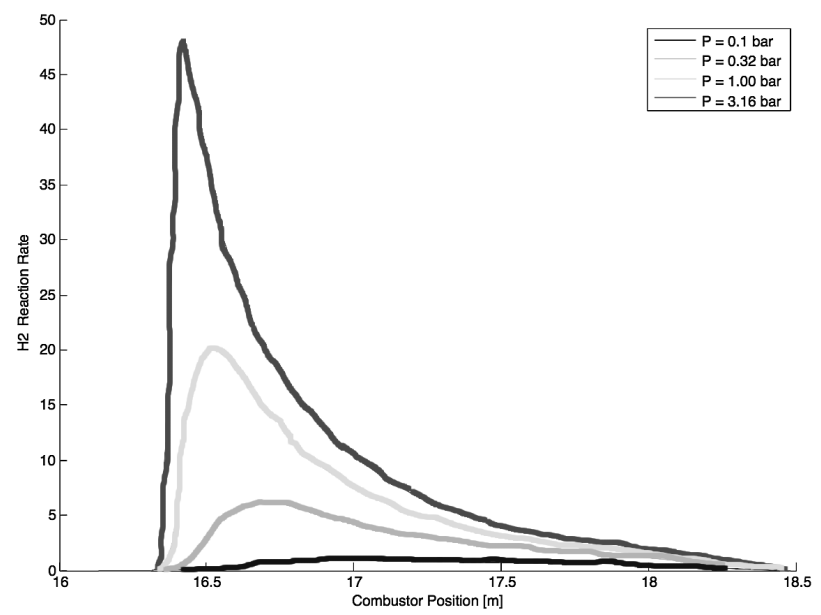

b)

Figure 4. (a) Contours of volumetric reaction rate of hydrogen $\left(\omega_{H 2}\right)$, and (b) the 1-D profiles of volumetric hydrogen reaction rate $\left(\bar{\omega}_{H 2}\right)$.

where $x_{C}$ is the axial distance from the injector, $d_{F}$ is the fuel jet diameter, and $R$ is the fuel jet momentum ratio $\left[\left(\rho_{F} U_{F}^{2}\right) /\left(\rho_{A} U_{A}^{2}\right)\right]^{1 / 2}$. Measurements also show that along the curved jet centerline, the mean fuel mass fraction $\left(Y_{F, C}\right)$ is:

$$
Y_{F, C}=c_{3}\left(\frac{\rho_{F}}{\rho_{A}}\right)^{1 / 3}\left(\frac{u_{F}}{u_{A}}\right)^{-1 / 3}\left(\frac{x_{C}}{d_{F}}\right)^{-2 / 3}
$$

The mean fuel mass fraction $\left(Y_{F}\right)$ also varies in the direction that is normal to the curved jet centerline; experiments show that this variation is nearly Gaussian in shape. Figure 4a shows the resulting contours of hydrogen mass fraction in an $\mathrm{x}-\mathrm{y}$ plane.

To account for 3-D turbulent mixing, the same mixing equations are used that appear in the CFD code FLUENT for an assumed-PDF model. At each (x, y, z) location in Figure 4a the mean mixture fraction is known, based on the empirical formulas stated above. Mixture fraction is related to the fuel mass fraction. Using the assumedPDF approach, fluctuations in mixture fraction are assumed to be proportional to the gradient in the mean mixture fraction. Another relation is used to compute the scalar dissipation rate from these fluctuations. As with the FLUENT approach, flamelet lookup tables are required to quantify the finite-rate chemistry. These were generated with the 
Stanford FLAMEMASTER code. This code considers strained flamelets and computes the volumetric reaction rate of hydrogen $\left(\omega_{H 2}\right)$ in moles $/ \mathrm{sec} / \mathrm{m}^{3}$ at each $(\mathrm{x}, \mathrm{y}, \mathrm{z})$ location in the jet in cross flow. Then these 3-D profiles are integrated to determine $\bar{\omega}_{H 2}$ from the following equation:

$$
\bar{\omega}_{H 2}(x)=\frac{1}{A} \iint \omega_{H 2}(x, y, z) d y d z
$$

Resulting profiles of $\bar{\omega}_{H 2}(x)$ appear in Figure $4 \mathrm{~b}$. This value is the react nrate that is required in equation (12). The final step is to compute the maximum fuel reaction rate $\left(R R_{F}\right)$ that is defined to be:

$$
R R_{F}=\left[\bar{\omega}_{H 2}(x)\right]_{\max }\left(\frac{W_{H 2}}{\rho_{H 2}}\right)
$$

The first term on the right $\left[\bar{\omega}_{H 2}(x)\right]_{\max }$ is the maximum value of the curve drawn in Figure $4 \mathrm{~b}$. Also appearing in equation (14) are the known values of the molecular weight of hydrogen $\left(W_{H 2}\right)$, which is $2.016 \mathrm{~g} / \mathrm{mole}$, and the density of the injected hydrogen $\left(\rho_{H 2}\right)$.

\section{Interpolation of Chemistry Tables using Proper Orthogonal Decomposition (POD)}

Sixteen large chemistry lookup tables were generated using the above approach. Each chemistry table corresponds to one of four different combustor inlet pressures and one of four inlet temperatures. Each table contains the reaction rates of eight major species as a function of mixture fraction and fluctuations in mixture fraction. Since the combustor inlet pressure and temperature usually falls in between the values and ranges of the generated lookup tables, a rapid interpolation method is required to determine correct reaction rates.

For such a large data set, standard interpolation methods between tables for pressures and temperatures were too slow or inaccurate, so the method of POD was applied and found to be rapid and accurate. The advantages and scalability of this technique are explored, and select comparisons are made of the full, non-reduced flamelet data to the results of the POD analysis.

The proper orthogonal decomposition (POD) is a well-defined method of producing reduced-order, but very accurate, models of large or complex data sets. It has been used in computational fluid dynamics (CFD) analysis to examine correlations of the structure of turbulent flowfields in time [28-30]. Other time-domain applications include constructing reduced-order models (ROM) of cylinder vortex shedding [31] and an aeroelastic model of a two-dimensional airfoil [32]. Analogous frequency-domain POD techniques have been explored by Kim [33], applied to a spring damper system and three-dimensional vortex lattice model. ROMs constructed using POD have been combined with structural dynamic models and applied to aeroelastic systems [34,35]; it has also been found effective for flutter analysis $[36,37]$. It has also been used for reduced-order models of atmospheric and oceanographic data, where the control space is high-dimensional [38]. However, in this work, the application of POD techniques to reduce multidimensional flamelet chemistry data used in a model for the mixing and combustion of turbulent jets in crossflow is explored.

Finally, using the developed POD architecture, various thermal properties and combustion efficiencies are calculated over a wide range of combustor operation conditions. As a hypersonic vehicle travels upward along an ascent trajectory, the static pressure in the scramjet combustor will decrease, which can lead to engine flameout. At low pressures the chemical reactions between the fuel and air become excessively slow. However, during ascent the flight Mach number is increasing. This increases the stagnation and static temperatures at the combustor entrance, tending to prevent flameout. These trends are observed and provide insight to determine flameout limits and ultimately flight trajectory envelopes for a hypersonic vehicle. Such results using simple and reduced models prove useful for rapid design and trajectory optimization.

\section{Application of the POD Method}

The combustion flamelet data used in MASIV are stored in large, multi-dimensional structures. In particular, the gas reaction rates are found in 3-D lookup tables for each gas species that contain the rate data for discrete permutations of mixture fraction $f$, mixedness $s$, and scalar dissipation $\chi$. Currently, data is retrieved through an interpolation of the function along the table near the given dimensions. Proper Orthogonal Decomposition (POD) presents a method of approximating the function with a linear combination of basis functions, which affords the same data to be reconstructed from a smaller data set and less storage space.

Consider a model where a vector $\mathbf{u}_{j}$ is calculated at $J$ discrete points within some domain for $j=1,2, \ldots, J$. The $\mathbf{u}_{j}$ vector may consist of $P$ quantities of interest as shown in equation (15).

For a two-dimensional inviscid flow problem, one might choose a vector $\mathbf{u}_{j}$ where $P=4$ and the states are density, $x$-momentum, $y$-momentum, and energy. But in general, $\mathbf{u}_{j}$ may contain any type of information for points $P$. In this 
work $\mathbf{u}_{j}=\dot{\omega}_{j}$, the reaction rate for a single species as a function of several flamelet reference variables, as described in Section III.

Combining the solution vectors $\mathbf{u}_{j}$ over the domain of $J$ points, we end up with a column vector of the form

$$
\mathbf{u}_{j}=\left(\begin{array}{c}
u_{j, 1} \\
\vdots \\
u_{j, P}
\end{array}\right), \quad \mathbf{q}^{m}=\left(\begin{array}{c}
\mathbf{u}_{1} \\
\vdots \\
\mathbf{u}_{J}
\end{array}\right)
$$

where $\mathbf{q}^{m}$ is a snapshot of the configuration for $m=1,2, \ldots, M$. Continuing the example of the inviscid CFD problem, $m$ can be chosen to be snapshots in time. However, $m$ may generally be any parameter or configuration affecting the solutions at each of the $J$ points.

The goal of POD is to represent all of the data approximately using a linear combination of $K \leq M$ basis vectors $\phi_{k}$. These vectors are much like snapshots, but are not in general equal to any individual snapshots. Linearly combining the $M$ snapshots yields

$$
\phi_{k}=\sum_{m=1}^{M} \mathbf{q}^{m} v_{k}^{m}=\mathbf{S} \mathbf{v}_{k} \Longleftrightarrow \Phi=\mathbf{S V}
$$

where $\mathbf{v}_{k}=\left(v_{k}^{1}, v_{k}^{2}, \cdots, v_{k}^{M}\right)^{T}$ and each entry is the contribution of the $m$ th snapshot to the $k$ th basis vector. $\Phi$ is a matrix of dimension $J \times K$ such that each colum is a basis vector $\phi_{k}$. S is a matrix of dimension $J \times M$ such that each column is a snapshot $\mathbf{q}^{m}$. V is a matrix of dimension $M \times K$ such that each column is $\mathbf{v}_{k}$.

Hall et al. [39] shows that this result reduces to an eigenvalue problem of the form

$$
\mathbf{S S}^{H} \mathbf{S v}_{k}=\lambda_{k} \mathbf{S v}_{k}
$$

where $\mathbf{S}^{H}$ is the Hermitian or conjugate transpose of $\mathbf{S}$. Solving for the eigenvalues $\lambda_{k}$ provides a correlation between the eigenvectors $\mathbf{v}_{k}$ and the basis vectors $\phi_{k}$. The eigenvectors with the largest values of $\lambda_{k}$ contribute the most to the values of $\phi_{k}$.

Rathinam and Petzold [40] explore the corollary for representing data sets in a general real space $\mathbb{R}^{n}$, using a subspace $\mathbb{S} \subset \mathbb{R}^{n}$. Here, POD minimizes the total square distance of the former data set to the projected data on $\mathbb{S}$, and $\mathbb{S}$ corresponds to the subspace determined by largest eigenvalues of the system.

\section{A. POD On Flamelet Chemistry Data}

The reduced-order mixing model examined in this work is part of the MASIV architecture, which is a complete flow model developed at the University of Michigan for a hypersonic vehicle with specified geometry and gas properties. It is further described in Torrez et al. [17] The mixing ROM incorporates the pressure, temperature, mean mixture fraction $f$, mixture fraction variance $s$, and the scalar dissipation rate $\chi$ of various gas species to determine the reaction rates throughout the combustion process and subsequently solve the $1 \mathrm{D}$ conservation equations. The reaction rate of each species varies with the aforementioned parameters, and those parameters are functions of the spatial variables in the vehicle combustor and isolator. As a result, multiple chemistry tables must be generated and stored to capture the reaction rate behavior for the wide range of pressures, temperatures, scalar dissipation rates, and species. The chemistry tables are then interpolated to find the particular reaction rate at a given location and condition.

Following the formulation described in Section II, the solution vector, or quantity of interest, $\mathbf{u}_{j}$ is set to be the reaction rate at the $j$ th value of the lookup variables. The mean mixture fraction and variance parameters are bounded between 0 and 1 , so the $j$-values are chosen to be discrete combinations of $f$ and $s$, respectively. The data sets contain $n_{1}=201$ discrete mean mixture fraction points and $n_{2}=25$ mixture fraction variance points, creating $J=n_{1} n_{2}=5025$ combinations of the aforementioned lookup variables. Therefore, one snapshot of the solution $\mathbf{q}^{m}$ in configuration $m$ is defined as

$$
\mathbf{u}_{j}=u_{j}=\dot{\omega}_{j}, \quad \mathbf{q}^{m}=\left(\begin{array}{c}
\dot{\omega}_{1} \\
\vdots \\
\dot{\omega}_{5025}
\end{array}\right), \quad \mathbf{S}=\left(\mathbf{q}^{1}, \cdots, \mathbf{q}^{M}\right)
$$

This snapshot is graphically shown in Figure 1, for a given configuration. $\mathbf{S}$ is then a row vector of the $M$ snapshots. For the simplest case in this work, these snapshots are chosen to be $M=46$ scalar dissipation rates at a fixed temperature and pressure for one species. 


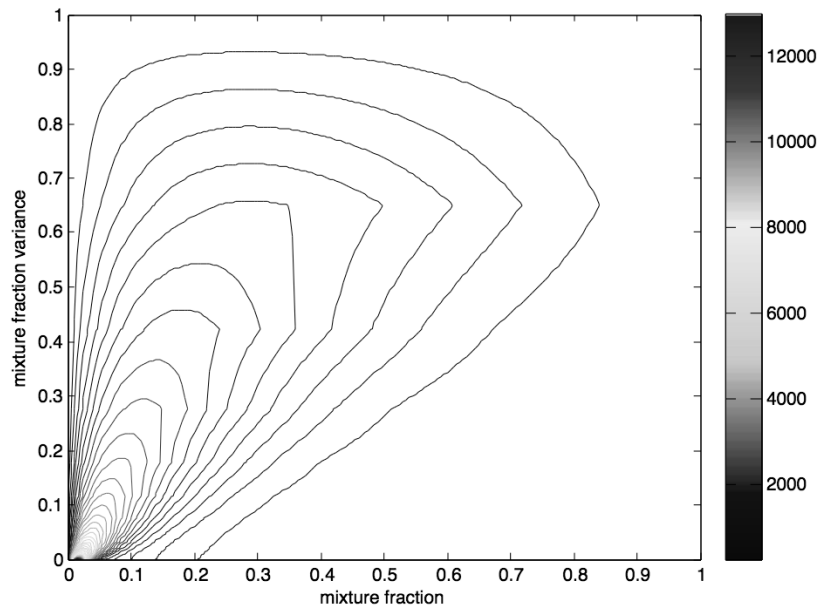

a) Contours of Reaction Rate

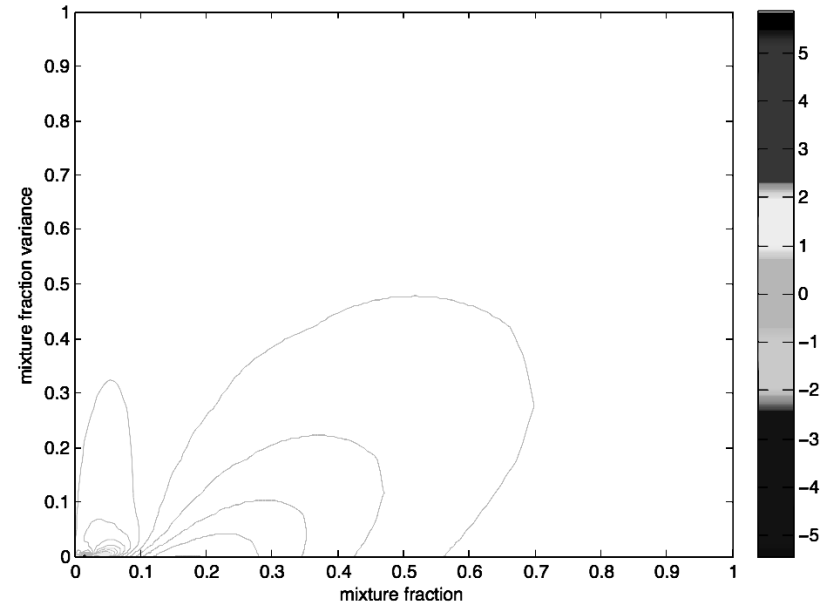

b) Contours of Reaction Rate Error

Figure 5. Flamelet conditions: $\mathrm{H} 2 \mathrm{O}$ at $\mathrm{p}=\mathbf{2 . 6 1} \mathrm{bar}, \mathrm{T}=1280 \mathrm{~K}$, and $\chi=312.3[1 / \mathrm{s}]$.

Discrete methods of POD are analogous to a matrix decomposition called singular value decomposition, which is a common mathematical tool implemented on many computational platforms, and is used for this analysis. Any matrix $\mathbf{S}$ can be written as the product

$$
\mathbf{S}=\mathbf{U} \Sigma \mathbf{W}^{H}
$$

where $\mathbf{U}, \mathbf{W}$ are unitary matrices of size $J$ and $M$, respectively. $\Sigma$ is a $J \times M$ matrix where the diagonal entries $\sigma_{m}=\sqrt{\lambda_{m}}$ are the square roots of the eigenvalues of $\mathbf{S}^{H} \mathbf{S}$, and are the only nonzero entries. For discrete POD analysis, we propose choosing a correlation quantity $0<\Gamma \leq 1$ and setting $K$ as the smallest integer such that

$$
\sum_{k=1}^{K} \sigma_{k} / \sum_{m=1}^{M} \sigma_{m} \geq \Gamma, \quad K \leq M
$$

for the $\Sigma$ matrix. The determination of a satisfying $K$ value subsequently yields the basis functions for the approximation as described in Section I. An analogous approach for the general real space example in [40] defines a correlation matrix from which the eigenvalues are determined. A similar procedure is followed in [38] for producing this matrix to compute the eigenvalues.

For this application, we look to the simplest case of taking the snapshots $\mathbf{q}^{m}$ of the $\mathbf{S}$ matrix in equation (18) to be a range of $M=46$ scalar dissipation rates for a single species, $\mathrm{H}_{2} \mathrm{O}$, at a fixed pressure and temperature. The correlation quantity is set to be $(1-\Gamma)=10^{-3}$. Decomposing $\mathbf{S}$ and satisfying the condition in equation (20) for $\Sigma$, one finds that $K=4$.

We create three new matrices: $\tilde{\Sigma}=\operatorname{diag}\left(\sigma_{1}, \cdots, \sigma_{K=4}\right)$, with all other entries being zero, and $\tilde{\mathbf{U}}, \tilde{\mathbf{W}}$ as the $(J \times 4)$ and $(M \times 4)$ parts of the $\mathbf{U}$ and $\mathbf{W}$ matrices, respectively. These three matrices approximate the function of the entire table with only a fraction of the data and storage. By multiplying $\tilde{\mathbf{U}}, \tilde{\Sigma}$, and $\tilde{\mathbf{W}}$ according to equation (19) we can recover a new data set $\tilde{\mathbf{S}}$, which is an accurate approximation of $\mathbf{S}$. A comparison of $\mathbf{S}$ and $\tilde{\mathbf{S}}$ for the same snapshot is shown in Figure 2.

The fractional savings can be measured by comparing the number of data points in the decomposition to that of the full set:

$$
\delta=\frac{(J \times K)+(K \times K)+(K \times M)}{(J \times M)} \times 100 \%
$$

For this example of $\mathrm{H}_{2} \mathrm{O}$ at $p=2.61$ bar and $T=1280 \mathrm{~K}$, we find that $\delta=8.78 \%$. This shows that a small percentage of the data can be retained, while still capturing nearly all of characteristics of the reaction rate behavior over the domain. 


\section{B. Additional Input Dimensions to the POD Approximation}

The POD analysis may be extended to account for additional parameters on which the reaction rate depends. In Section III, the gas species, pressure, and temperature were specified such that the only variable parameter outside of the $J$ points of mixture fraction/variance was the scalar dissipation rate. The range of the scalar dissipation rates made up the $M$ snapshots. Say that we are now interested in the reaction rates for $N$ number of species. We must then obtain tabulated data for the matrix $\mathbf{S}^{n}$ given in equation (18) for each of the $N$ species. Defining a new matrix $\mathbf{S}_{\text {total }}$, a concatentation of the $\mathbf{S}$ matrices, we get

$$
\mathbf{S}_{\text {total }}=\left(\mathbf{S}^{1}, \cdots, \mathbf{S}^{N}\right)
$$

Introducing additional dimensions to the data follows the same procedure. Allowing pressure as a variable will require the result in equation (22) to be tabulated at each pressure. Accounting for temperature variation again multiplies the size of the resulting matrix by the number of temperature points, and so on. Thus, the general $\mathbf{S}_{\text {total }}$ matrix will be of the form:

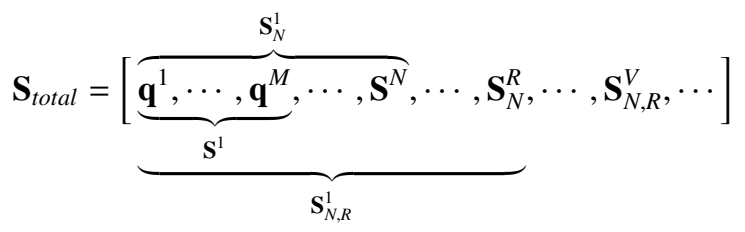

for additional dimensions with number of points $M, N, R, V, \ldots$, respectively. Note that while the total number of snapshots $\mathbf{q}^{M \times N \times R \times V \times \cdots}$ increases with the added dimensions, the number of $J=5025$ points remains the same.

Table 1 compares the results of POD for increasing numbers of snapshots and dimensions, for the same correlation quantity $(1-\Gamma)=10^{-3}$.

Table 1. Scaling POD to Higher Dimensional Data Sets

\begin{tabular}{lccc}
\hline \hline Varying Parameters & \# Data Points & \# Basis & Fraction $\delta$ \\
\hline None (1) - no reduction & 5025 & 1 & $100 \%$ \\
$\times$ Scalar Dissipation (46) & 231150 & 4 & $8.78 \%$ \\
$\times$ Species (8) & 1849200 & 8 & $2.34 \%$ \\
$\times$ Pressures (4) & 7396800 & 10 & $0.75 \%$ \\
\hline \hline
\end{tabular}

The information here shows that POD is highly effective in approximating multidimensional data sets, while only adding few eigenvalues/basis functions for each new parameter and using a smaller fraction of the total data.

\section{Model Accuracy}

To determine the accuracy of the POD approximation, we compare the original data set $\mathbf{S}$ to the recovered data set $\tilde{\mathbf{S}}=\tilde{\mathbf{U}} \tilde{\Sigma} \tilde{\mathbf{W}}^{H}$ after the matrix decomposition and eigenvalue analysis. Integrating the absolute error of the reaction rate at each point in the matrices is an insufficient approach, as larger data sets will inevitably sum to larger total errors. Instead, we look to match the aggregate properties of both data sets, the mean, variance, and standard deviation, and infer the accuracy of the approximation from their likeness. Table 2 shows the percent difference of the root-meansquare $(\tilde{\mu}, \mu)$, variance $(\tilde{v}, v)$, and standard deviation $(\tilde{\sigma}, \sigma)$ of the approximated data set $\tilde{\mathbf{S}}$ with respect to $\mathbf{S}$, for each case discussed in Section IV.

Table 2. Mean, Variance, and Standard Deviation Error

\begin{tabular}{lccc}
\hline \hline Varying Parameters & $(\tilde{\mu} / \mu-1)$ & $(\tilde{v} / \nu-1)$ & $(\tilde{\sigma} / \sigma-1)$ \\
\hline None (1) - no reduction & $-3.8 \times 10^{-8}$ & $5.8 \times 10^{-5}$ & $2.9 \times 10^{-5}$ \\
$\times$ Scalar Dissipation (46) & $-8.8 \times 10^{-8}$ & $8.0 \times 10^{-6}$ & $4.0 \times 10^{-6}$ \\
$\times$ Species (8) & $-9.2 \times 10^{-8}$ & $-1.8 \times 10^{-7}$ & $-9.2 \times 10^{-8}$ \\
$\times$ Pressures (4) & $-8.5 \times 10^{-8}$ & $-1.7 \times 10^{-7}$ & $-8.5 \times 10^{-8}$ \\
\hline
\end{tabular}

The results show that even while the size of the data increases significantly with each added dimension, the aggregate behavior of the reaction rate for both the original data set and the POD approximation remain strongly correlated. 
It is important, however, to recognize that this is not necessarily true for the general case. It is possible to construct data sets such that the matrix $\mathbf{S}$ becomes ill-conditioned and the eigenvalue analysis produces less accurate basis vectors. In such cases, POD is not sufficient and other methods must be used for a better approximation.

We can see that for this application, using POD to reduce and approximate large flamelet chemistry data sets proves to be very effective and accurate. Where the multidimensionality of the reaction rate requires numerous chemistry tables to be generated, POD is most useful in reducing the storage and memory footprint, while maintaining the integrity of the data with low error margins. This allows those computational resources to be allocated elsewhere in the reduced-order model. If not all of the data is needed, the relevant portions can be recovered by combining only parts of the decomposition, eliminating the wasted storage of keeping the full chemistry tables. Recovering the full data set is easily done by multiplying the full matrices from the decomposition.

\section{Flameout Limits During Ascent}

To understand the flameout limit curve, the following simple examples are presented for an idealized hypersonic vehicle. The critical Damkohler number at flameout is the constant $D a_{H}^{*}$, which must be determined from experiment. From Ozawa [1], the chemical reaction rate is represented as:

$$
R R=C_{2}\left[\frac{T}{300 K}\right]^{n}\left[\frac{p_{3}}{1 a t m}\right]^{m}\left[\frac{S_{L, 0}}{1 m / s}\right]^{2}
$$

where the constants $C_{2}, n$ and $m$ must be determined from experimental flameout measurements. The factor $\left(S_{L, 0} / 1 \mathrm{~m} / \mathrm{s}\right)^{2}$ in equation (24) accounts for the different types of fuel that may be used. Each fuel has a known value of the laminar burning velocity $S_{L, 0}$ which is measured at stoichiometric conditions (pressure at $1 \mathrm{~atm}$, temperature at $298 \mathrm{~K}$ ). From the definition of Damkohler number in equations (2-4), and equation (24), it follows that at flameout:

$$
U_{3}^{*}=H\left(\frac{C_{2}}{D a_{H}^{*}}\right)\left[\frac{T}{300 K}\right]^{n}\left[\frac{p_{3}}{1 a t m}\right]^{m}\left[\frac{S_{L, 0}}{1 m / s}\right]^{2}
$$

For an idealized vehicle, the following assumptions are made. The adjustable inlet is designed to provide a fixed value of Mach number at the combustor entrance $\left(M_{3}\right)$ equal to 2 , and a fixed inlet pressure ratio $p_{3} / p_{a}$ equal to 20 . The inlet is adiabatic so the stagnation temperature $T_{0,3}$ equals $T_{0, a}$, which is $T_{a}\left(1+0.2 M_{\infty}^{2}\right)$. The ambient static temperature $T_{a}$ does not change significantly with altitude for a short ascent. The left side of equation (25) is the Mach number times the speed of sound at station 3 , so:

$$
U_{3}^{*}=M_{3}\left[\gamma R\left(\frac{T_{3}}{T_{0,3}}\right)\right]^{1 / 2}\left(T_{0, a}\right)^{1 / 2}
$$

With the above assumptions, $M_{3},\left(T_{3} / T_{0,3}\right)$, and $T_{a}$ are constant during ascent. So we obtain:

$$
U_{3}^{*}=C *\left(1+0.2 M_{\infty}^{2}\right)^{1 / 2}
$$

where $\mathrm{C}$ is some constant. The right side of equation (25) becomes the following, under the same assumptions:

$$
U_{3}^{*}=C *\left(1+0.2 M_{\infty}^{2}\right)^{n}\left(p_{a}\right)^{m}
$$

Now the ambient static pressure varies inversely with altitude (h), and combining equations (27) and (28) leads to:

$$
\begin{gathered}
\left(1+0.2 M_{\infty}^{2}\right)^{1 / 2}=C *\left(1+0.2 M_{\infty}^{2}\right)^{n}\left(p_{a}\right)^{m} \\
h=C\left(1+0.2 M_{\infty}^{2}\right)^{(2 n-1) /(2 m)}
\end{gathered}
$$

Ozawa's data shows that $n=2$ and $m=1$, so the flameout limit curve is determined to be the following relationship between altitude $h$ and flight Mach number:

$$
h=C\left(1+0.2 M_{\infty}^{2}\right)^{3 / 2}
$$

This is the result of the simple scaling analysis. It yields the flameout limit curve in Figure 2 that is drawn as a dotted line. The many assumptions made in the scaling analysis can be replaced by computations using the MASIV inlet and combustor codes. This more accurate exercise will be reported in a future paper. 


\section{Acknowledgements}

This research is funded by the Air Force Research Laboratory/Air Vehicles Directorate grant FA8650-07-2-3744 that has supported the Michigan/AFRL Collaborative Center in Control Sciences. Dr. Michael Bolender is the technical monitor. The first author also has been supported by a University of Michigan Fellowship. The authors are grateful for the technical assistance provided by Dr. Derek Dalle and Dr. Sean Torrez.

\section{References}

[1] Ozawa, R. I., "Survey of Basic Data on Flame Stabilization and Propagation for High Speed Combustion Systems," The Marquart Co., TR AFAPL-TR-70-81, 1971.

[2] BAXTER, M. R. and LEFEBVRE, A. H., "Flame stabilization in high-velocity heterogeneous fuel-air mixtures,” Nov. 1992.

[3] Niioka, T., Kobayashi, H., Hasegawa, S., and Terada, K., "Flame stabilization characteristics of strut divided into two parts in supersonic airflow," Journal of Propulsion and Power, Vol. 11, No. 1, 1995, pp. 112-116.

[4] Plee, S. and Mellor, a., "Characteristic time correlation for lean blowoff of bluff-body-stabilized flames," Combustion and Flame, Vol. 35, 1979, pp. 61-80.

[5] Kundu, K. M., Banerjee, D., and Bhaduri, D., "Theoretical Analysis on Flame Stabilization by a Bluff-Body," Combustion Science and Technology, Vol. 17, No. 3-4, 1977, pp. 153-162.

[6] Shanbhogue, S. J., Husain, S., and Lieuwen, T., "Lean blowoff of bluff body stabilized flames: Scaling and dynamics," Progress in Energy and Combustion Science, Vol. 35, No. 1, 2009, pp. 98-120.

[7] Driscoll, J. F. and Rasmussen, C. C., "Correlation and Analysis of Blowout Limits of Flames in High-Speed Airflows," Journal of Propulsion and Power, Vol. 21, No. 6, Nov. 2005, pp. 1035-1044.

[8] Gruber, M. R., Donbar, J. M., Carter, C. D., and Hsu, K.-Y., "Mixing and Combustion Studies Using Cavity-Based Flameholders in a Supersonic Flow," Journal of Propulsion and Power, Vol. 20, No. 5, 2004, pp. 769-778.

[9] Gruber, M. R., Baurle, R. a., Mathur, T., and Hsu, K.-Y., "Fundamental Studies of Cavity-Based Flameholder Concepts for Supersonic Combustors," Journal of Propulsion and Power, Vol. 17, No. 1, 2001, pp. 146-153.

[10] Rasmussen, C. C., Driscoll, J. F., Hsu, K. Y., Donbar, J. M., Gruber, M. R., and Carter, C. D., "Stability limits of cavitystabilized flames in supersonic flow," Proceedings of the Combustion Institute, Vol. 30 II, No. 2, Jan. 2005, pp. $2825-2833$.

[11] Rasmussen, C. C. and Driscoll, J. F., "Characteristics of Cavity- Stabilized Flames in a Supersonic Flow," Journal of Propulsion and Power, Vol. 21, No. 2, 2005, pp. 765-768.

[12] Zhang, T., Wang, J., Qi, L., Fan, X., and Zhang, P., "Blowout Limits of Cavity-Stabilized Flame of Supercritical Kerosene in Supersonic Combustors," Journal of Propulsion and Power, Vol. 30, No. 5, 2014, pp. 1-6.

[13] Bolender, M. a. and Doman, D. B., "Nonlinear Longitudinal Dynamical Model of an Air-Breathing Hypersonic Vehicle," Journal of Spacecraft and Rockets, Vol. 44, No. 2, 2007, pp. 374-387.

[14] Parker, J. T., Serrani, A., Yurkovich, S., Bolender, M. a., and Doman, D. B., "Control-Oriented Modeling of an Air-Breathing Hypersonic Vehicle,” Journal of Guidance, Control, and Dynamics, Vol. 30, No. 3, 2007, pp. 856-869.

[15] Dalle, D. J., Torrez, S. M., Driscoll, J. F., Bolender, M. a., and Bowcutt, K. G., "Minimum-Fuel Ascent of a Hypersonic Vehicle Using Surrogate Optimization," Journal of Aircraft, April 2014, pp. 1-14.

[16] Dalle, D. J., Fotia, M. L., and Driscoll, J. F., "Reduced-Order Modeling of Two-Dimensional Supersonic Flows with Applications to Scramjet Inlets," Journal of Propulsion and Power, Vol. 26, No. 3, May 2010, pp. 545-555.

[17] Torrez, S. M., Driscoll, J. F., Ihme, M., and Fotia, M. L., "Reduced-Order Modeling of Turbulent Reacting Flows with Application to Ramjets and Scramjets," Journal of Propulsion and Power, Vol. 27, No. 2, March 2011, pp. $371-382$.

[18] Torrez, S. M., Dalle, D. J., and Driscoll, J. F., "New Method for Computing Performance of Choked Reacting Flows and Ram-to-Scram Transition," Journal of Propulsion and Power, Vol. 29, No. 2, March 2013, pp. 433-445.

[19] Dalle, D. J., Torrez, S. M., and Driscoll, J. F., "Rapid Analysis of Scramjet and Linear Plug Nozzles," Journal of Propulsion and Power, Vol. 28, No. 3, 2012, pp. 545-555.

[20] O’Neill, M. K. L. and Lewis, M. J., “Design tradeoffs on scramjet engine integrated hypersonic waverider vehicles,” Journal of Aircraft, Vol. 30, No. 6, 1993, pp. 943-952.

[21] Bowcutt, K. G., "Multidisciplinary Optimization of Airbreathing Hypersonic Vehicles," Journal of Propulsion and Power, Vol. 17, No. 6, 2001, pp. 1184-1190.

[22] McQuade, P. D., Eberhardt, S., and Livne, E., "CFD-based aerodynamic approximation concepts optimization of a twodimensional scramjet vehicle," Journal of Aircraft, Vol. 32, No. 2, 1995, pp. 262-269.

[23] Starkey, R. P. and Lewis, M. J., "Critical Design Issues for Airbreathing Hypersonic Waverider Missiles," Journal of Spacecraft and Rockets, Vol. 38, No. 4, 2001, pp. 510-519. 
[24] Tarpley, C. and Lewis, M. J., "Stability derivatives for a hypersonic caret-wing waverider," Journal of Aircraft, Vol. 32, No. 4, 1995, pp. 795-803.

[25] O', T. F., Brien, Starkey, R. P., and Lewis, M. J., "Quasi-One-Dimensional High-Speed Engine Model with Finite-Rate Chemistry," 2001.

[26] Chavez, F. R. and Schmidt, D. K., “Analytical aeropropulsive-aeroelastic hypersonic-vehicle model with dynamic analysis," Journal of Guidance, Control, and Dynamics, Vol. 17, No. 6, 1994, pp. 1308-1319.

[27] Hasselbrink, E. F. and Mungal, M. G., “Transverse jets and jet flames. Part 2. Velocity and OH field imaging,” 2001.

[28] Lumley, J. L., "The Strucure of Inhomogeneous Turbulent Flows," Atmospheric Turbulence and Radio Wave Propagation, 1967, pp. 166-178.

[29] Berkooz, G., Holmes, P., and Lumley, J. L., "The Proper Orthogonal Decomposition in the Analysis of Turbulent Flows," Annual Review of Fluid Mechanics, Vol. 25, No. 1, 1993, pp. 539-575.

[30] Poje, A. C. and Lumley, J. L., "A model for large-scale structures in turbulent shear flows," Journal of Fluid Mechanics, Vol. 285, No. -1, 1995, pp. 349.

[31] Tang, K., Graham, W., and Peraire, J., “Active flow control using a reduced order model and optimum control," AIAA paper, , No. June, 1996.

[32] Romanowski, M., "Reduced order unsteady aerodynamic and aeroelastic models using Karhunen-Loeve eigenmodes," AIAA paper, 1996, pp. 7-13.

[33] Kim, T., "Frequency-domain Karhunen-Loeve method and its application to linear dynamic systems," AIAA Journal, Vol. 36, No. 11, 1998, pp. 2117-2123.

[34] Lucia, D. J., Beran, P. S., and Silva, W. a., "Aeroelastic System Development Using Proper Orthogonal Decomposition and Volterra Theory," Journal of Aircraft, Vol. 42, No. 2, 2005, pp. 509-518.

[35] Beran, P. and Pettit, C., "Prediction of nonlinear panel response using proper orthogonal decomposition," AIAA paper, , No. c, 2001.

[36] Thomas, J. P., Dowell, E. H., and Hall, K. C., “Three-Dimensional Transonic Aeroelasticity Using Proper Orthogonal Decomposition-Based Reduced-Order Models," Journal of Aircraft, Vol. 40, No. 3, 2003, pp. 544-551.

[37] Kim, T., Hong, M., Bhatia, K. G., and Sengupta, G., "Aeroelastic Model Reduction for Affordable Computational Fluid Dynamics-Based Flutter Analysis," AIAA Journal, Vol. 43, No. 12, 2005, pp. 2487-2495.

[38] Cao, Y., Zhu, J., Navon, I. M., and Luo, Z., "A reduced-order approach to four-dimensional variational data assimilation using proper orthogonal decomposition,” International Journal for Numerical Methods in Fluids, Vol. 53, No. 10, April 2007, pp. 1571-1583.

[39] Hall, K. C., Thomas, J. P., and Dowell, E. H., "Proper orthogonal decomposition technique for transonic unsteady aerodynamic flows," AIAA Journal, Vol. 38, No. 10, 2000, pp. 1853-1862.

[40] Rathinam, M. and Petzold, L. R., “A New Look at Proper Orthogonal Decomposition,” SIAM Journal on Numerical Analysis, Vol. 41, No. 5, Jan. 2003, pp. 1893-1925. 